\title{
Psychotic Disorder due to the Use of Marijuana, Dextromethorphan, and Caffeine: A Case Report and Review of the Literature
}

\author{
Yoseva Hotnauli, Muhammad Surya Husada*(D) \\ Department of Psychiatry, Faculty of Medicine, Universitas Sumatera Utara, Medan, Indonesia
}

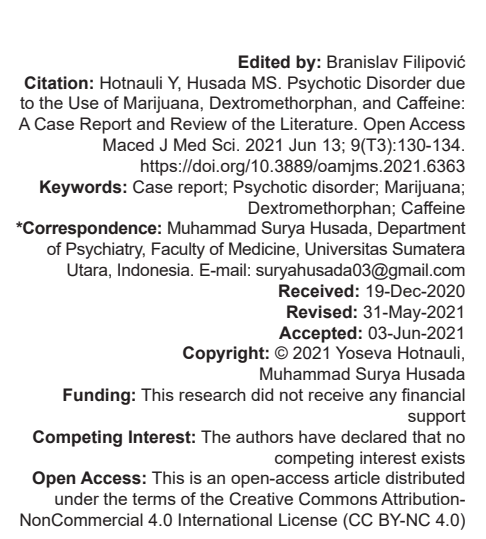

\begin{abstract}
BACKGROUND: Drug abuse occurs widely in various parts of the world. The drugs that are misused are not only cocaine, or heroin, but also drugs that are commonly prescribed. If you want to get drunk, usually use dextromethorphan (DMP) pills, but now switch to mixing herbal cough medicines with energy drinks. Herbal cough medicine is usually used as a cough reliever, but in teens today, herbal cough medicine is starting to be abused. Mixing energy drinks using herbal cough medicine are motivated by a lack of positive activities or activities, influenced by peers or the environment where the subject lives, lack of application of religious and school education, lack of the role of parents in supervising their children, easy access to herbal cough medicines and drinks energy in large quantities, and the price is cheap. The crime that occurred is not only limited to theft but also drugs and drinking. The only difference, they are not drunk with liquor, but mixed energy drinks with some herbal cough medicine sachets.

CASE REPORT: We found a case of acute psychotic disorders due to cannabis use and other psychoactive substances, namely, a mixture of herbal cough medicine containing DMP and energy drinks containing caffeine, after 2 days of use, in a 20 -year-old male, found a delusion and hallucinations.

CONCLUSIONS: Every use of addictive substances and psychotropic substances will have an impact on life and health. The use of addictive and psychotropic substances when consumed in excess of the dose will damage some organ functions, affect the smooth operation of organ systems, and can also cause acute psychosis.
\end{abstract}

\section{Introduction}

A cluster of psychotic phenomena that occur during or immediately after psychoactive substance use and are characterized by vivid hallucinations (typically auditory, but often in more than one sensory modality), misidentifications, delusions and/or ideas of reference (often of a paranoid or persecutory nature), psychomotor disturbances (excitement or stupor), and an abnormal affect, which may range from intense fear to ecstasy. The sensorium is usually clear but some degree of clouding of consciousness, though not severe confusion, may be present. A psychoactive substance-induced psychotic disorder may present with varying patterns of symptoms. These variations will be influenced by the type of substance involved and the personality of the user [1].

\section{Marijuana}

The term "marijuana" usually refers to the preparation of tobacco leaves and flowers from the Cannabis sativa plant [2]. Delta-9-tetrahydrocannabinol (THC) is a cannabinoid which is primarily responsible for the psychoactive effects on cannabis. Common names for Cannabis are marijuana, grass, pot, weed, tea, and Mary Jane [3]. Different ways of using cannabis make absorption, metabolism, and excretion of THC different. If cannabis is smoked, THC is absorbed from the lungs into the bloodstream in minutes. This is the first metabolism in the lung, then in the liver. 9-carboxyTHC metabolite can be detected in the blood within minutes after smoking. The peak THC level in the blood is usually reached within 10 min of smoking and drops by around $5-10 \%$ from the initial level within $1 \mathrm{~h} \mathrm{[4].}$

According to the World Health Organization, marijuana is the most widely prohibited substance cultivated in the world, traded, and misused. About $2.5 \%$ of the world's population (147 million people) use it. Its use is more common among men than gender pleasing women that widened from 2007 to 2014. Its use is widespread in the population of adolescents and young adults [2].

The initial state of acute poisoning defines the symptoms enjoyed by recreational users: Euphoria, changes in perception such as time and spatial distortion, intensification of ordinary sensory experiences, and motor disorders. Not all effects of marijuana poisoning are welcomed by users because of some experience, unpleasant psychological reactions such as panic, fear, or depression. Acute intoxication also affects the heart and vascular system, resulting in Cannabis-induced 
tachycardia and postural hypotension. Central nervous system (CNS) and respiratory depression have been recorded with high doses in animal models. Studies show that inhaled doses of 2-3 milligrams $(\mathrm{mg}) \mathrm{THC}$ and ingested doses of 5-20 mg THC can cause attention, memory, executive function, and short-term memory problems. Cannabis poisoning can cause acute psychosis in many individuals and can produce short-term exacerbations of pre-existing psychotic diseases such as schizophrenia. Psychiatric symptoms observed in several studies included depersonalization, fear of death, irrational panic, and paranoid ideas [2].

Cannabinoids can be detected in the urine, head hair, pubic hair, sweat, urine, saliva, and blood from their use. In some cases, it can be detected in urine more than 11 weeks after use. Blood THC levels can range between 0 and $500 \mathrm{mg} / \mathrm{ml}$ depending on the potential of cannabis and the time since smoking [5].

\section{Dextromethorphan (DMP)}

DMP is an ordinary cough medicine that is sold freely in pharmacies, but who would have thought it turned out to be a very disturbing parent, especially in the field of education. One example of cough medicines that are sold freely in pharmacies is herbal cough medicine. The herbal cough medicine is a cough medicine (each $7 \mathrm{~mL}$ sachet contains DMP $15 \mathrm{mg}$ ). The problem is that this herbal cough medicine has been widely abused by teenagers, ranging from teenagers to adults. It is done to get the effect of being jazzed and is considered to make people calm, can relieve stress. In the environment, the DMP drug education has become well-known among students, not because it is a cough medicine but is known as a drug that can make people fly or cause euphoria and a sense of calm when used in large enough doses [6].

First introduced in the market in the 1950s in America, DMP is a cough suppressant drug that is very popular and has been freely available and is often found in cough and cold medicines. The indication for this drug is for dry cough or cough without phlegm. The dosage for adults is $10-20 \mathrm{mg}$ orally every $4 \mathrm{~h}$ or $30 \mathrm{mg}$ every $6-8 \mathrm{~h}$ with a maximum dose of $120 \mathrm{mg} / \mathrm{day}$. The dosage of children aged $6-12$ years is $5-10 \mathrm{mg}$ orally every $4 \mathrm{~h}$ or $15 \mathrm{mg}$ every $6-8 \mathrm{~h}$ with a maximum dose of $60 \mathrm{mg} /$ day. For ages $2-6$ years, the dose is $2.5-5 \mathrm{mg}$ orally every $4 \mathrm{~h}$ or $7.5 \mathrm{mg}$ or every $6-8 \mathrm{~h}$ with a maximum dose of $30 \mathrm{mg} /$ day. The anti-cough effect can last 5-6 h after oral use. If used according to the rules, this drug is relatively safe, rarely causing significant side effects. The most common side effect is drowsiness [7].

DMP is a morphine derivative compound, which has the chemical name/IUPAC (+) - 3-methoxy17-methyl-( $9 \alpha, 13 \alpha, 14 \alpha)$-morphinan, a dextrose isomer of levomethorphan. DMP is an N-Methyl D-aspartate (NMDA) receptor antagonist which is in the CNS. Thus, the pharmacological effects of DMP, especially if at high doses, resemble phencyclidine or ketamine which are NMDA receptor antagonists. Antagonism of NMDA receptors can cause euphoric effects, antidepressants, and the effects of psychosis such as visual or auditory hallucinations. Supported easily obtained, and the price is low, this is what causes DMP to be a drug that is often misused in high doses. The misuse of this DMP has been quite extensive and has now reached a worrying stage, and this is what "forces" BPOM to announce its withdrawal from the market. In California (USA), the abuse of DMP began to bloom in the 2000s. The use of high doses of DMP is not without problems. Apart from providing behavioral effects, DMP intoxication or overdose can cause hyperexcitability, fatigue, sweating, chaotic speech, hypertension, and bulging eyes (nystagmus) [7].

In terms of behavioral effects, DMP abusers describe a dose-dependent four plateau effects, as follows [6] (Table 1):

Table 1: Dextromethorphan abuser a dose-dependent four plateau effect

\begin{tabular}{lll}
\hline Plateau & Dose $(\mathrm{mg})$ & Behavioral effects \\
\hline $1^{\text {st }}$ & $100-200$ & Mild stimulation \\
$2^{\text {nd }}$ & $200-400$ & Euphoria and hallucinations \\
$3^{\text {rd }}$ & $300-600$ & Impaired visual perception and loss of motor coordination \\
$4^{\text {th }}$ & $500-1500$ & Dissociative sedation \\
\hline
\end{tabular}

Judging from the use of this drug, if it is used in the dosage according to the doctor's recommendation, it is not dangerous for people who consume it, but if the use is beyond the doctor's recommendation or in high doses, this is very dangerous to the health of one's soul, and this is included in the category of misuse of cough medicine DMP. In addition to the trigger factors why DMP is misused above, misusers usually consume DMP to get effects similar to the use of ketamine. Although ketamine is a drug that is used as a general anesthetic (anesthesia) so that the side effects caused include confusion, conditions such as dreams, a sense of personal identity loss, speech and movement disorders, disorientation, drowsiness, and even continue to faint [6].

\section{Caffeine}

Caffeine (1,3,7-trimethylxanthine, guaranine) is a type of alkaloid and psychostimulant plant found in tea leaves, coffee, cocoa beans, and cola beans. Caffeine, theophylline, theobromine, and paraxanthine are routinely detected in toxicological samples due to exposure to caffeine diets. An average cup of coffee or tea in the United States is reported to contain between 40 and $150 \mathrm{mg}$ of caffeine even though specialty coffee may contain much higher doses. Caffeine experiences rapid absorption in oral administration. About $90 \%$ of ingested doses are absorbed from the stomach in 20 min, with peak plasma concentrations occurring around 50-60 min later [8].

Caffeine is now increasingly consumed in a more concentrated form as "shot" energy (5 h extra 
energy 207 mg per 2-ounce container), energy drinks, or, most of the attention, like caffeine $100 \%$ anhydrous, high doses given by yourself drink addictive [8]. Caffeine includes stimulants or stimulants that if consumed will provide a stimulating effect on the user, this is actually what attracts the use of drinks containing caffeine. One example of energy drinks is "kratingdaeng." In one bottle of "kratingdaeng" drink, there are $50 \mathrm{mg}$ of caffeine. Some of the stimulating effects obtained after consuming caffeine within normal limits are reducing fatigue and reducing drowsiness [9]. The fatal dose of acute oral caffeine in adult humans is estimated to be in the range of $150-200 \mathrm{mg} / \mathrm{kg}$ body weight [8]. Caffeine is a mild CNS stimulant. Insufficient doses, however, it can result in flushing, shivering, agitation, irritability, loss of appetite, weakness, and tremor. Hypertension, hypotension, tachycardia, vomiting, fever, delusions, hallucinations, seizures, arrhythmias, cardiac arrest, coma, and death have been reported in cases of overdose [10].

It has been known for over 40 years that substances such as hallucinogens, stimulants, and cannabis in high doses can be associated with or possibly cause psychotic states. These drugs affect the dopaminergic and glutaminergic systems in the brain, which have both been associated with psychotic symptoms such as hallucinations and delusions. However, psychotic symptoms induced by substances generally tend to be short-lived in comparison with psychosis in schizophrenia, and the presentation is slightly different, with predominating agitation and confusion in psychosis following drug use [11].

Many people with mental health issues use substances, and in people with psychosis, problematic drinking and use of illicit drugs occur more frequently than in the general population. Several drugs of misuse can lead to psychotic reactions that are unequivocally a direct consequence of the drug taken. In such cases, the drug is usually taken in large or repeated doses and the psychotic reaction is manifest shortly afterward, often after only a few hours. People with severe mental illness and substance misuse are less likely to recover from a psychotic episode and more likely to experience a relapse. Rates of relapse in psychosis are also higher among those who misuse other drugs, especially stimulants [11].

\section{Case Presentation}

A 20-year-old man who did not have a significant psychiatric history comes to an emergency installation (ER) with erratic and disturbing behavior. He was at home, angry, and throwing things around him. He said that he was a famous artist and he was afraid that people would steal something from him and that "something bad" would happen, sometimes, he also said that he was a "prophet" sent by God to heal all those who were sick in this world. His parents reported that he had insomnia, thoughts of racing, and euphoria over the past week.

After arriving at the ER, vital signs are at temperature of $36.8^{\circ} \mathrm{C}$, heart rate $80 / \mathrm{min}$, blood pressure $130 / 80 \mathrm{mmHg}$, and respiratory rate $20 / \mathrm{min}$. The ocular examination was notable for mydriasis (symmetrically reactive to light) and ocular clonus. The skin felt warm and dry and mucous membranes were dry. Patellar and Achilles deep tendon reflexes were increased compared with deep tendon reflexes on the upper extremities. Bilateral inducible sustained ankle clonus, lasting approximately $1 \mathrm{~min}$, was noted. He was difficult to be directed, and his mental status shows an unstable mood and that is inappropriate. The process of thinking the patients was loose and irregular with thoughts that block. He was paranoid, hyper-religious, and supports auditory hallucinations. He denied the idea of suicide or murder. His attention and memory were considered disturbed even though they were not formally tested, but on psychiatric examination using Positive and Negative Syndrome Scale, his total score was 116.

For presentations of acute psychosis, emergency medical providers carry out a comprehensive examination to exclude the organic etiology of psychosis or concomitant medical morbidity. The result of the electrocardiogram was normal sinus rhythm with normal pulse frequency $(84 \times /$ minute). The toxicological results of urine were positive for THC more than $500 \mathrm{ng} / \mathrm{mL}$; the toxicological results of urine were negative for cocaine, amphetamines, benzodiazepines, and opioids. A B12 level was elevated at $1166 \mathrm{pg} / \mathrm{mL}$. Laboratory results for complete blood tests are within normal limits. Patients claimed to use marijuana cigarettes and consumed a mixture of herbal cough medicines containing DMP and energy drinks containing caffeine. Where "komix" cough medicine containing 20 DMP sachets and "kratingdaeng" energy drinks containing five cans of caffeine. He used marijuana for the past 2 weeks but used the other mixture in the past 2 days. He cannot measure his consumption how many times a day. He believes the paranoid onset and insomnia coincides with last week's consumption of THC. Patients refused to use alcohol recently. He denied that there was a fall or a history of traumatic brain injury as well as recognition from his parents confirming this history. A history of family mental illness was not found.

Because the patient was hyperactive, disturbing, enters another patient's room, and touches the other patient, then he was directed safely and physically restrained gently for the safety of patients and others. He was placed in a particular room for psychiatric emergency services for his safety and further evaluation and treatment. He was given $2 \mathrm{mg}$ Risperidone $2 \times 1 / 2$ tab;ets orally for the management of psychosis 
Twenty-four hours after further observation, his psychotic symptoms and anxiety persisted, he said that his food was poisoned and asked whether he was still a prophet or not. The patient did not know where he was currently living. Her awareness and attention were intact.

Forty-eight hours after continued observation, paranoia and hallucinations of patients had improved slowly. The patient could reflect his paranoid ignorance and "strange thoughts." With better insight, he confirmed the use of many THC products used, in addition to the consumption of "komix" drugs containing DMP and kratingdaeng energy-containing drinks, which are difficult to quantify. Patients were diagnosed with psychotic disorders that induced marijuana use and the use of other psychoactive substances (a mixture of DMP and caffeine), patients were instructed to follow up with outpatient mental health to ensure completion of their psychosis and start treatment for substance abuse.

\section{Discussion}

The acute onset of symptoms with marijuana use, high serum marijuana metabolite levels, and symptomatic resolution with detoxification suggest that these symptoms were secondary to marijuana use. Marijuana increases the risk of myocardial infarction within $1 \mathrm{~h}$ of use, and cardiovascular events have been reported in otherwise healthy patients. In this case, an elevated B12 level was found on the workup of the patient's psychiatric symptoms and suspected to have been caused by energy drink consumption; only later did the patient confirm this suspicion. By its effects on mesolimbic dopamine activity, caffeine may precipitate psychosis, exacerbate chronic psychosis, or worsen affective lability and mood states. This patient's high THC metabolite level, this patient the toxicological results of urine were positive for THC more than $500 \mathrm{ng} / \mathrm{mL}$ demonstrates that oral administration can achieve high serum THC levels and suggests a doseresponse relationship between serum metabolite levels and the severity of psychosis [2], [5], [8].

There is no single explanation for the high level of association between psychosis and substance misuse. These two disorders are usually regarded as separate diagnostic entities and therefore satisfy the strict criteria for comorbid disorders: The presence of "any distinct clinical entity that has existed or that may occur during the clinical course of a patient who has the index disease understudy." Although neither substance misuse nor schizophrenia is uncommon, the frequency with which they present together is many times higher than would be expected by chance. It is far from clear why this is so, but several theories have been put forward for the association [11]:
1. Substance misuse either precipitates the onset of, or is a direct cause of, psychosis.

2. Substance misuse is a common consequence of a psychotic disorder.

3. There is a common cause, or vulnerability, to both substance misuse and psychosis.

In making a diagnosis of a disorder, it is necessary to pay attention to the diagnostic criteria of the disorder whether it meets the criteria or not, in this case, diagnostic criteria are used, namely, from the International Classification of Diseases $10^{\text {th }}$ Revision (ICD-10).

Psychotic disorders that occur during or immediately after drug use (usually within $48 \mathrm{~h}$ ) should be noted here unless the condition is not a manifestation of a withdrawal condition with delirium (F1x.4) or slow onset. Late-onset psychotic disorders (with an onset of more than 2 weeks after substance use) can occur but must be classified into F1x.75. Psychotic disorders caused by psychoactive substances can appear with varied patterns. This variation will be influenced by the type of substance used and the user's personality. Drug-induced psychotic disorders are generally closely associated with high doses and prolonged substance use [1].

Furthermore, it is necessary to avoid mistakes in psychosis as a more severe condition (e.g. schizophrenia), even though psychoactive substances cause the diagnosis of psychosis. Many psychotic conditions caused by psychoactive substances are short as long as there are no more drugs used [1].

Based on the case report above, after complete psychiatric history taking, clinical interviews, and mental status examinations, psychotic disorders that occur during or immediately after drug use in this patient meet the diagnostic criteria in ICD-10 [1].

\section{Conclusions}

The relationship between psychosis and coexisting substance misuse is complex. People with psychosis and coexisting substance misuse often have poor physical health. There is no single explanation for the high level of association between psychosis and substance misuse. These two disorders are usually regarded as separate diagnostic entities and therefore satisfy the strict criteria for comorbid disorders: The presence of "any distinct clinical entity that has existed or that may occur during the clinical course of a patient who has the index disease understudy." However, the use of addictive and psychotropic substances, when consumed more than the dosage, will damage some organ functions, affect the smooth functioning of the organ system, and also cause acute psychosis disorders. 


\section{Declarations}

\section{Authors' contributions}

Patient was recruited by $\mathrm{YH}$ and $\mathrm{MSH}$. All authors have contributed to and have approved the final manuscript.

\section{Acknowledgments}

We sincerely thank to participant and all those have contributed to this article.

\section{Ethic approval and consent to participate}

All procedures were approved by the ethics committee Department of Psychiatry, Faculty Medicine, University of Sumatera Utara Hospital. Participant received verbal and written descriptions of the study and provided their written informed consent.

\section{Consent for publication}

Written informed consent for publication of their clinical details was obtained from the patient. A copy of the consent form is available for review by the editor of this journal.

\section{Availability of data and materials}

The datasets used and/or analyzed during the current study are available from the corresponding author on reasonable request.

\section{References}

1. World Health Organization, ICD-10 Version; 2016. p. 79-80.

2. Turner AR, Aqrawal S. Marijuana toxicity. In: Stat Pearls. Treasure Island, FL: Stat Pearls Publishing; 2021.

3. Sadock BJ, Sadock VA. Cannabis-related disorder. In: Kaplan and Sadock's Synopsis of Psychiatry Behavioral Science and Clinical Psychiatry. $11^{\text {th }}$ ed. Philadelphia, PA: Lippincott Williams and Wilkins; 2015. p. 1385-94.

4. Tasman A, Kay J Lieberman JA, First MB, Riba MB Psychiatry. $4^{\text {th }}$ ed. Chichester: John Wiley and Sons, Ltd.; 2015. p. 1458-61.

5. Hall WD, Degenhardt L. Cannabis-related disorders. In: Sadocks BJ, Sadock VA, editors. Kaplan and Sadock Comprehensive Textbook of Psychiatry. $10^{\text {th }}$ ed. New York: Wolters Kluwers; 2017. p. 3358-73.

6. World Health Organization. Dextromethorphan Pre-Review Report. $35^{\text {th }}$ ed. Geneva: Expert Committee on Drug Dependence; 2012. p. 1-26.

7. Chyka AP, Erdman RA, Manoguerra SA, Christianson G, Booze LL, Nelson LS, et al. Dextromethorphan poisoning: An evidence-based consensus guideline for out-of-hospital management. Clin Toxicol. 2007;45(6):662-77. https://doi. org/10.1080/15563650701606443

PMid:17849242

8. Jabbar SB, Hanly MG. Fatal caffeine overdose: A case report and review of literature. Am J Forensic Med Pathol. 2013;34(4):321-4. https://doi.org/10.1097/ paf.0000000000000058

PMid:24196726

9. Muhyi M. Effect of supplemental drinks, electrolyte drinks and normal water on sports performance. Adiraga Sci J. 2015;1(1):69-90.

10. Kerrigan S, Lindsey T. Fatal caffeine overdose: Two case reports. Forensic Sci Int. 2005;153(1):67-9. https://doi.org/10.1016/j. forsciint.2005.04.016

PMid: 15935584

11. National Collaborating Centre for Mental Health. Psychosis with Coexisting Substance Misuse: Assessment and Management in Adults and Young People. Leicester, UK: British Psychological Society; 2011. 